\title{
Evaluation of Stopping Power Ratio Calculation Using Dual-energy Computed Tomography With Fast Kilovoltage Switching for Treatment Planning of Particle Therapy
}

\author{
SHINGO OHIRA ${ }^{1,2}$, YASUHIRO IMAI ${ }^{3}$, YUHEI KOIKE ${ }^{4}$, SHUNSUKE ONO ${ }^{1}$, YOSHIHIRO UEDA $^{1}$, \\ MASAYOSHI MIYAZAKI ${ }^{1}$, MASAHIKO KOIZUMI ${ }^{2}$ and KOJI KONISHI ${ }^{1}$ \\ ${ }^{1}$ Department of Radiation Oncology, Osaka International Cancer Institute, Osaka, Japan; \\ ${ }^{2}$ Department of Medical Physics and Engineering, Osaka University Graduate School of Medicine, Osaka, Japan; \\ ${ }^{3}$ CT Engineering, GE Healthcare Japan Corporation, Tokyo, Japan; \\ ${ }^{4}$ Department of Radiology, Kansai Medical University, Osaka, Japan
}

\begin{abstract}
Background/Aim: This study evaluated the calculation accuracy of the stopping power ratio (SPR) using dual-energy computed tomography with fast kilovoltage switching (FKSCT) for particle therapy. Materials and Methods: A tissue characterization phantom with various reference materials was scanned to obtain single-energy computed tomography (SECT) images and generate virtual monochromatic images at $77 \mathrm{keV}\left(\mathrm{VMI}_{77 \mathrm{keV}}\right)$ and $140 \mathrm{keV}$ $\left(V M I_{140 \mathrm{keV}}\right)$, water density (WD) images, and effective $Z\left(Z_{\text {eff }}\right)$ images. For SECT, VMI $77 \mathrm{keV}$ and VMI $140 \mathrm{keV}$ lookup tables were generated to convert the measured Hounsfield value into the theoretical SPR for a normal phantom size. Subsequently, the reference materials were scanned in small and large phantoms. The SPR was calculated using the lookup tables of $\operatorname{SECT}\left(S P R_{S E C T}\right)$ images, $V M I_{77 \mathrm{keV}}\left(S P R_{77 \mathrm{keV}}\right)$, and VMI $140 \mathrm{keV}$ $\left(S P R_{140 \mathrm{keV}}\right)$, and it was derived from the $W D$ and $Z_{\text {eff }}$ $\left(S P R_{W D}\right)$. Results: In the normal-sized phantom, the overall mean difference between $S P R_{W D}$ and theoretical SPR was $-0.3 \%$, and remained below $2 \%$ for most reference materials. For the large phantom, the overall mean absolute difference for $\operatorname{SPR}_{140 \mathrm{keV}}(3.0 \%, p=0.006)$ and $\operatorname{SPR} R_{W D}(3.2 \%, p=0.002)$ for the reference materials was significantly lower than that for $S P R_{S E C T}(5.9 \%)$. For the small phantom, a significant reduction in the mean difference in the SPR calculation was
\end{abstract}

This article is freely accessible online.

Correspondence to: Shingo Ohira, Ph.D., Department of Radiation Oncology, Osaka International Cancer Institute, 3-1-69 Otemae, Chuo-ku, Osaka 537-8567, Japan. Tel: +81 669451181, Fax: +81 669451900, e-mail: oohira-si@mc.pref.osaka.jp

Key Words: Stopping power ratio, dual-energy CT, fast kilovoltage switching, calculation accuracy, particle therapy. observed in $\operatorname{SPR}_{77 \mathrm{keV}}(1.0 \%, p=0.001)$ and $\operatorname{SPR}_{140 \mathrm{keV}}(1.1 \%$, $p=0.013)$ compared with $S P R_{S E C T}(2.2 \%)$. Conclusion: $V M I_{140 \mathrm{keV}}$ generated using FKSCT significantly improves the estimation accuracy of SPR compared with SECT. Thus, FKSCT may be used to improve the dose calculation accuracy for treatment planning of particle therapy.

In recent years, particle therapy using proton or carbon ion beams has been increasingly used for cancer treatment owing to its superior dose distributions and higher biological effect compared with conventional photon beam radiotherapy (1). Thus, particle therapy can improve the tumor control probability, while reducing the probability of normal tissue complications. Charged particle beams travel a finite distance within the human body depending on the beam energy according to the Bragg peak, while photon beams travel through the entire body. As particle range prediction based on computed tomography (CT) numbers (in Hounsfield unit-HU) presents uncertainty, strict safety margins are used in clinical practice (2).

For dose calculation during treatment planning of particle therapy, the HU values are converted into stopping power ratios (SPRs) relative to water using a lookup table (LUT) (3). However, the accuracy of the HU values measured using conventional single-energy CT (SECT) is affected by various factors, such as the scanning parameters, presence of highdensity materials, and size of the scanned object (4). Dualenergy CT (DECT) has enabled the generation of virtual monochromatic images (VMIs), which are expected to reduce the uncertainty in the measurement of HU values because VMIs obtained using high-energy imaging are less affected by the abovementioned factors (5). Accordingly, the use of VMIs can improve the accuracy of SPR calculation. Moreover, DECT using dual-layer detectors can be used to calculate the electron density relative to water $\left(\varrho_{\mathrm{e}}\right)$ and 
in vivo $36: 103-110(2022)$

Table I. Specifications and measured values for reference materials of tissue characterization phantom.

\begin{tabular}{|c|c|c|c|c|c|c|c|c|}
\hline \multirow[b]{2}{*}{$\begin{array}{l}\text { Rod number } \\
\text { \# }\end{array}$} & \multirow[b]{2}{*}{$\begin{array}{c}\text { Reference } \\
\text { materials }\end{array}$} & \multicolumn{5}{|c|}{ Measured value } & \multicolumn{2}{|c|}{ Theoretical value } \\
\hline & & $\begin{array}{c}120 \mathrm{kVp} \\
(\mathrm{HU})\end{array}$ & $\begin{array}{c}77 \mathrm{keV} \\
(\mathrm{HU})\end{array}$ & $\begin{array}{c}140 \mathrm{keV} \\
(\mathrm{HU})\end{array}$ & $\begin{array}{l}\text { Water density } \\
\qquad(\mathrm{mg} / \mathrm{ml})\end{array}$ & Effective Z & Electron density & Effective Z \\
\hline 1 & LN-300 Lung & -686.2 & -690.3 & -701.9 & 293.9 & 7.77 & 0.278 & 7.55 \\
\hline 2 & LN-450 Lung & -519.8 & -527.6 & -533.8 & 463.9 & 7.64 & 0.455 & 7.53 \\
\hline 3 & AP6 Adipose & -82.4 & -72.5 & -58.9 & 945.8 & 6.69 & 0.932 & 6.21 \\
\hline 4 & BR-12 Breast & -28.4 & -33.1 & -33.5 & 966.4 & 7.54 & 0.960 & 6.93 \\
\hline 5 & Water Insert & 2.6 & 4.5 & -4.1 & 993.3 & 7.91 & 1.000 & 7.48 \\
\hline 6 & CT Solid Water 1 & 4.0 & 8.2 & -1.6 & 995.1 & 7.95 & 0.987 & 7.74 \\
\hline 7 & CT Solid Water 2 & 7.7 & 4.4 & -4.0 & 993.2 & 7.94 & 0.987 & 7.74 \\
\hline 8 & CT Solid Water 3 & 8.7 & 1.5 & -6.3 & 991.0 & 7.91 & 0.987 & 7.74 \\
\hline 9 & CT Solid Water 4 & -0.8 & -4.2 & -2.4 & 998.3 & 7.46 & 0.987 & 7.74 \\
\hline 10 & BRN-SR2 Brain & 34.7 & 43.0 & 32.7 & $1,029.5$ & 7.94 & 1.047 & 6.09 \\
\hline 11 & LV1 Liver & 66.8 & 66.6 & 65.4 & $1,065.2$ & 7.57 & 1.060 & 7.74 \\
\hline 12 & IB Inner bone & 223.9 & 209.9 & 112.7 & $1,079.4$ & 10.42 & 1.096 & 10.42 \\
\hline 13 & B200 Bone mineral & 229.6 & 215.0 & 122.0 & $1,090.0$ & 10.32 & 1.104 & 10.42 \\
\hline 14 & $\mathrm{CB} 2-30 \% \mathrm{CaCO} 3$ & 430.3 & 417.6 & 293.8 & $1,251.3$ & 10.62 & 1.277 & 10.90 \\
\hline 15 & $\mathrm{CB} 2-50 \% \mathrm{CaCO} 3$ & 782.4 & 761.6 & 512.1 & $1,426.4$ & 12.05 & 1.469 & 12.54 \\
\hline 16 & SB3 Cortical Bone & 1174.2 & 1170.3 & 766.5 & $1,627.8$ & 13.13 & 1.694 & 13.64 \\
\hline
\end{tabular}

effective atomic number $\left(Z_{\text {eff }}\right)$. Based on these values, the SPR can be directly calculated without using a LUT (6). DECT-based SPR calculation has also been demonstrated using dual-spiral acquisition (7). Therefore, DECT seems promising to improve the accuracy of dose calculation for treatment planning of particle therapy.

In DECT using fast kilovoltage switching (FKSCT), simultaneous projections at two energies and almost the same location can be acquired, and projection-based reconstruction enables beam hardening correction (8). However, the VMIs generated from FKSCT are more susceptible to beam hardening, which leads to inaccurate HU estimation compared with that using SECT images (5). Although FKSCT can directly provide accurate $Z_{\text {eff }}(9), \varrho_{e}$ cannot be calculated. Instead, FKSCT generates a water density image (WDI) (in $\mathrm{mg} / \mathrm{ml}$ ) that has a linear relation with $\varrho_{\mathrm{e}}$. Thus, the WDI can be used for SPR calculation (10). Nevertheless, to the best of our knowledge, the SPR calculation accuracy based on VMIs and WDIs using FKSCT has not been evaluated.

This study consisted of two parts. First, we generated three types of LUTs based on SECT, VMI at $77 \mathrm{keV}$ $\left(\mathrm{VMI}_{77 \mathrm{keV}}\right.$, equivalent to SECT), and VMI at $140 \mathrm{keV}$ $\left(\mathrm{VMI}_{140 \mathrm{keV}}\right)$ and evaluated the SPR calculation based on WDI and $Z_{\text {eff }}$ obtained using FKSCT. Second, we investigated the robustness of the abovementioned SPR calculation methods for different phantom sizes.

\section{Materials and Methods}

Phantom imaging. Ethical approval was not required because our study design only included the use of phantoms. Image acquisition was performed using FKSCT (Revolution HD; GE Medical Systems, Milwaukee, WI, USA) and the following scanning parameters: tube voltage of $80 / 140 \mathrm{kVp}$, tube current of $600 \mathrm{~mA}$, volume CT dose index of $32.5 \mathrm{mGy}$, and gantry rotation of $1 \mathrm{~s}$ per rotation for DECT, and $120 \mathrm{kVp}, 400 \mathrm{~mA}, 32.6 \mathrm{mGy}$, and $1 \mathrm{~s}$ per rotation as the respective parameters for SECT. Reconstruction was applied to the acquired data using a slice thickness of $2 \mathrm{~mm}$ and a field of view of $500 \mathrm{~mm}$. Image acquisition was repeated five times to minimize random variations, and the acquired images were transferred to a workstation (AdvantageSim; GE Medical Systems).

A tissue characterization phantom (Gammex RMI 467; Gammex RMI, Middleton, WI, USA) containing the tissue-mimicking reference materials listed in Table I was used for imaging. The theoretical value of the electron density for each reference material was provided by the manufacturer. According to the compositions of reference materials provided by Landry et al. (11), the theoretical $Z_{\text {eff }}$ in Table I was calculated as follows:

$$
Z_{e f f}=\sqrt[\beta]{\sum_{i}^{n} \frac{\omega_{i} Z_{i}}{A_{i}} Z_{i}^{\beta} / \sum_{i}^{n} \frac{\omega_{i} Z_{i}}{A_{i}}}
$$

where $\beta$ is set to $3.3, i$ is the number of elements composing a reference material, $Z_{i}$ is the atomic number of element $i, A_{i}$ is the mass of the element, and $w_{i}$ represents the composition. Then, the theoretical SPR was calculated based on the theoretical $\varrho_{\mathrm{e}}$ and $Z_{\text {eff }}$ values as follows (12):

$$
S P R=\rho_{e} \times \frac{12.77-\left(a \times Z_{e f f}+b\right)}{8.45},(2)
$$

where $a$ and $b$ are 0.125 and 3.378 for reference materials with $Z_{\text {eff }} \leq 8.5$, respectively, and 0.098 and 3.376 for $Z_{\text {eff }}>8.5$, respectively. 


\section{LUT method}
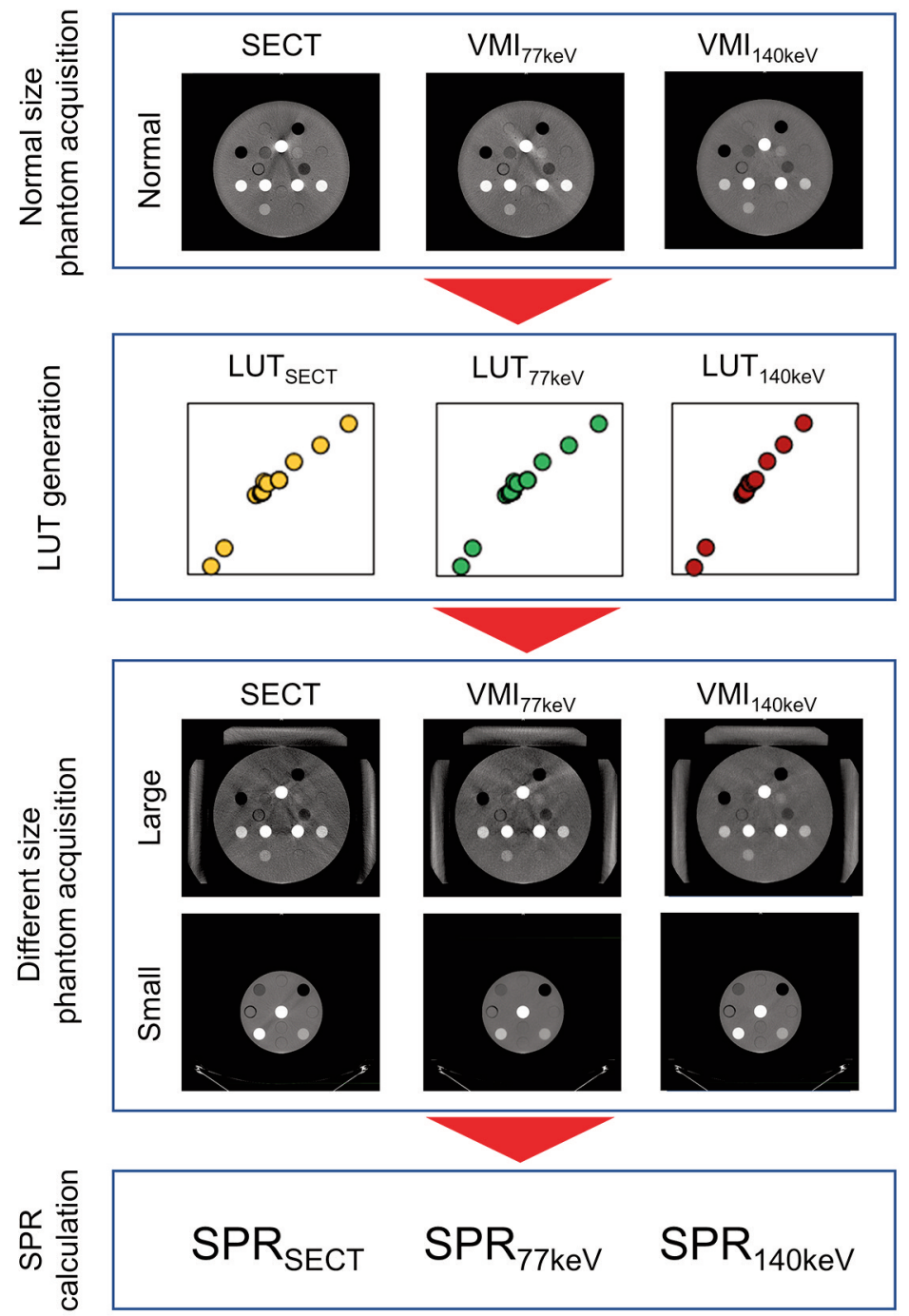

\section{Direct method}

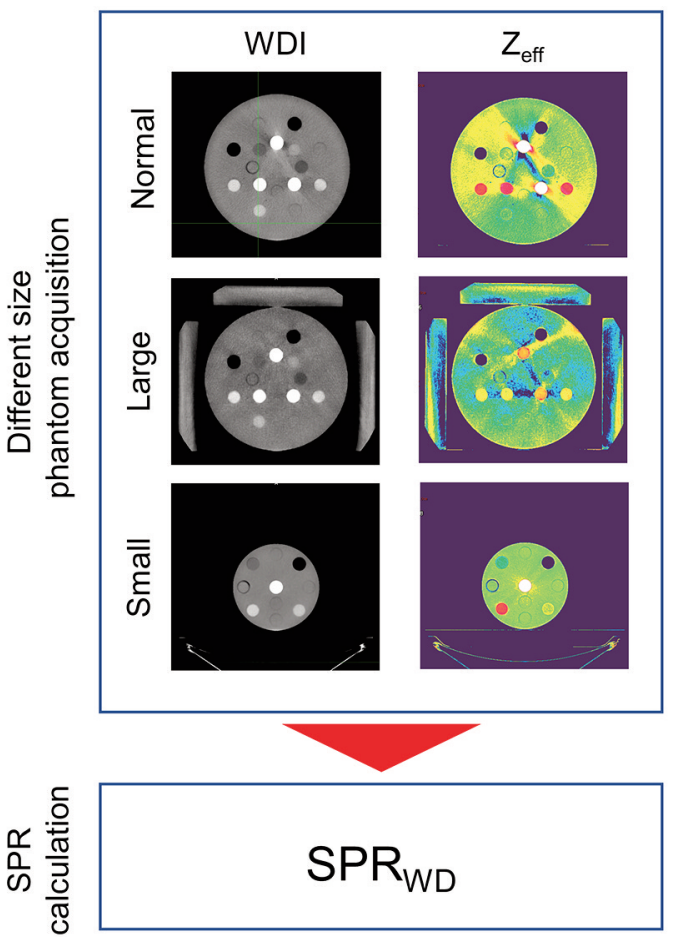

Figure 1. Calculation of stopping power ratio (SPR) based on single-energy computed tomography (SECT), virtual monochromatic images at 77 $\mathrm{keV}\left(\mathrm{VMI}_{77 \mathrm{keV}}\right)$ and $140 \mathrm{keV}\left(\mathrm{VMI}_{140 \mathrm{keV}}\right)$, water density image $(\mathrm{WDI})$, and effective $Z\left(Z_{\text {eff }}\right)$ image.

SPR calculation using LUTs. Figure 1 shows a diagram for SPR calculation. $\mathrm{VMI}_{77 \mathrm{keV}}$ and $\mathrm{VMI}_{140 \mathrm{keV}}$ were reconstructed from DECT data. For the quantitative analysis, a circular region of interest with a diameter of $20 \mathrm{~mm}$ was set at the center of the reference materials. The LUTs for SECT $\left(\mathrm{LUT}_{\mathrm{SECT}}\right), \mathrm{VMI}_{77 \mathrm{keV}}$ $\left(\mathrm{LUT}_{77 \mathrm{keV}}\right)$, and $\mathrm{VMI}_{140 \mathrm{keV}}\left(\mathrm{LUT}_{140 \mathrm{keV}}\right)$ used to convert the HU values into theoretical SPRs were obtained.

The reference materials were then scanned for two phantom sizes to investigate the robustness of SPR calculation. Specifically, three solid water phantoms $\left(300 \times 300 \times 50 \mathrm{~mm}^{3}\right)$ were arranged around a tissue characterization phantom to establish a large phantom. In addition, the reference materials were inserted into the cylindrical region (180 $\mathrm{mm}$ in diameter) of a multi-energy phantom (Gammex RMI 1472; Gammex RMI) to establish a small phantom.
The HU values of the reference materials were measured for the two phantom sizes. In the LUT method, the SPRs $\left(\mathrm{SPR}_{\mathrm{SECT}}\right.$, $S P R_{77 \mathrm{keV}}$, and $\mathrm{SPR}_{140 \mathrm{keV}}$ ) of each reference material for the two phantom sizes were obtained from the measured HU values using the corresponding LUTs.

SPR calculation based on WDI and $Z_{\text {eff. }}$ For the normal, large, and small phantoms, the WDI and $Z_{\text {eff }}$ were reconstructed, and the quantitative values for each material were measured using a circular region of interest. Using FKSCT, the water density (in milligrams per cubic centimeter, $\mathrm{mg} / \mathrm{cm}^{3}$ ) instead of $\varrho_{\mathrm{e}}$ and the measured $Z_{\text {eff }}$ were used to obtain the SPR as follows:

$$
S P R=\text { water density } \times \frac{12.77-\left(a \times Z_{e f f}+b\right)}{8.45},
$$



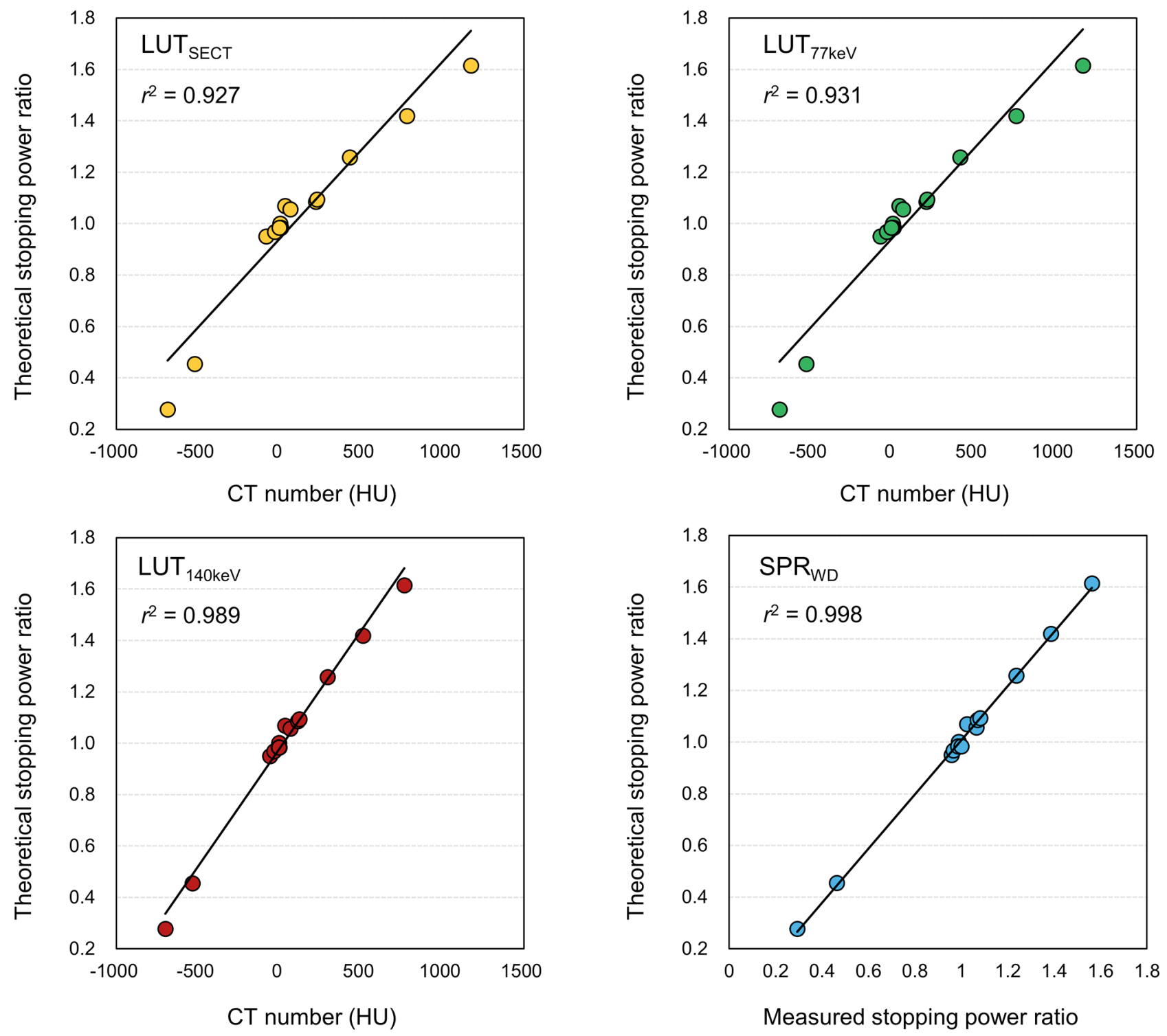

Figure 2. Lookup tables (LUT) generated based on SECT (LUT $\left.T_{S E C T}\right), V M I_{77 \mathrm{keV}}\left(L U T_{77 \mathrm{keV}}\right)$ and $V M I_{140 \mathrm{keV}}\left(L U T_{140 \mathrm{keV}}\right)$, and relationship between theoretical and measured SPR calculated using WDI (SPR $\left.R_{W D}\right)$.

where $a$ and $b$ are the same as in the theoretical SPR calculation (Equation 2). As $Z_{\text {eff }}$ for reference materials with low $\varrho_{\mathrm{e}}$ (rods 1 and 2 in Table I) could not be measured using the AdvantageSim workstation, the $Z_{\text {eff }}$ values of such reference materials were manually determined using the monochromatic attenuation ratio method (9). First, the ratio of the linear attenuation coefficients at 70 and $120 \mathrm{keV}$ for a theoretical $Z_{\text {eff }}$ (obtained from the NIST XCOM database) was plotted (13). Then, the ratio of the measured linear attenuation coefficients at 70 and $120 \mathrm{keV}$ was converted into the corresponding $Z_{\text {eff }}$ value.

Data analysis. To validate the accuracy of SPR calculation based on WDI and $Z_{\text {eff }}$, the percentage difference between the measured $\left(\mathrm{SPR}_{\mathrm{WD}}\right)$ and theoretical $\mathrm{SPR}$ values was obtained for each reference material. Then, the absolute difference between the measured $\left(\mathrm{SPR}_{\mathrm{SECT}}, \mathrm{SPR}_{77 \mathrm{keV}}, \mathrm{SPR}_{140 \mathrm{keV}}\right.$, and $\left.\mathrm{SPR}_{\mathrm{WD}}\right)$ and theoretical SPRs for the large and small phantoms was used to evaluate the calculation robustness. The statistical accuracy differences between the SPR calculations based on DECT and SECT scans was determined using paired Wilcoxon signed-rank tests (SPSS software, version 27; IBM, Armonk, NY, USA). Statistical significance was indicated by $p<0.05$.

\section{Results}

The values for the reference materials in the tissue characterization phantom used to generate the LUTs are listed 


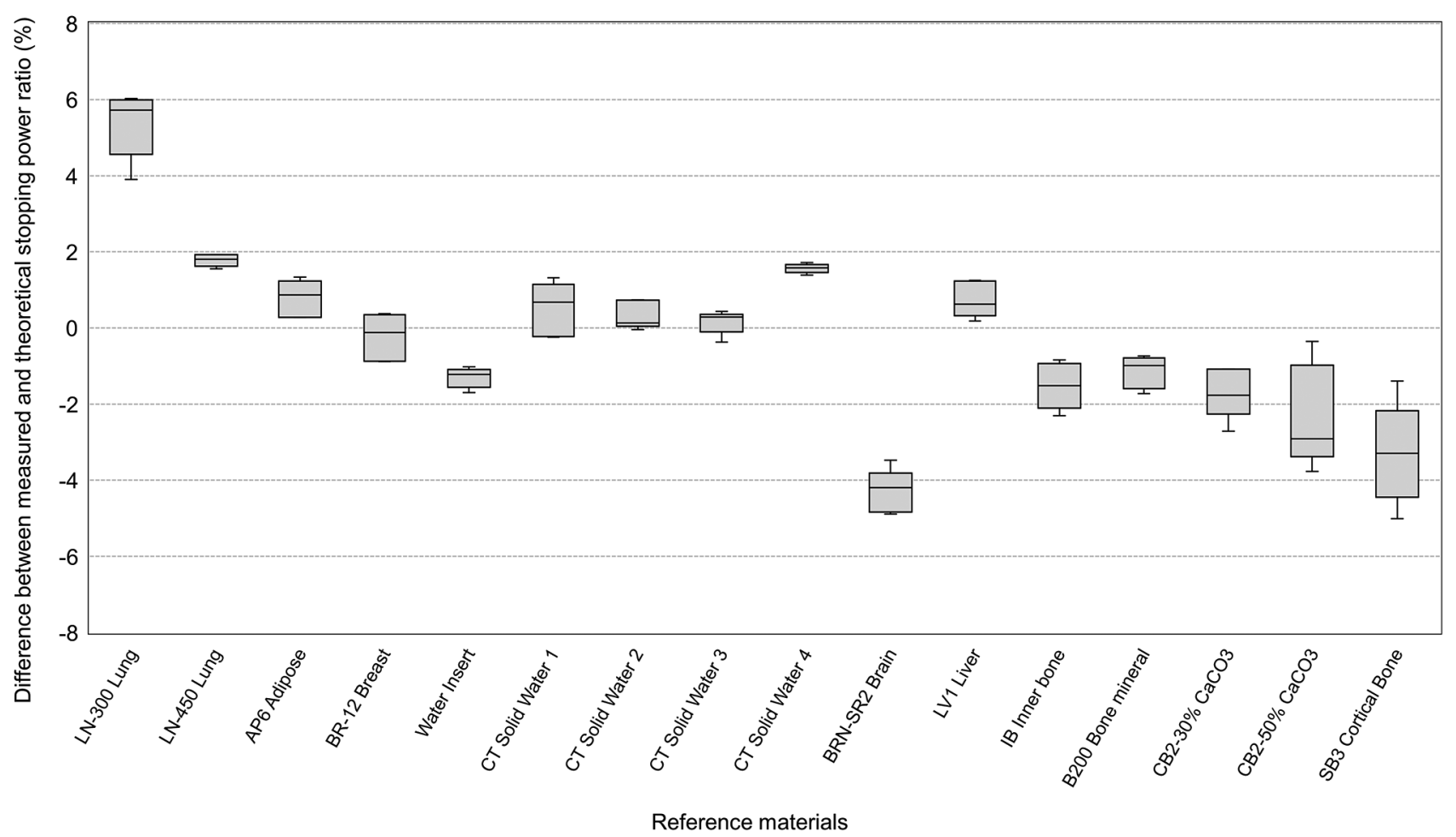

Figure 3. Percentage difference between stopping power ratio calculated based on water density image and $Z_{\text {eff }}$ and theoretical SPR for each reference material.

in Table I. The HU values in SECT and $\mathrm{VMI}_{77 \mathrm{keV}}$ were almost the same for each material, while those in $\mathrm{VMI}_{140 \mathrm{keV}}$ were smaller, especially for high-density materials $(1,174.2$, $1,170.3$, and $766.5 \mathrm{HU}$ in SECT, $\mathrm{VMI}_{77 \mathrm{keV}}$, and $\mathrm{VMI}_{140 \mathrm{keV}}$ for SB3 cortical bone, respectively). The measured water density proportionally increased with the electron density. The difference between the measured and theoretical $Z_{\text {eff }}$ was below 9\%, except for BRN-SR2 brain (difference of 30\%). The relations between the measured values and theoretical SPRs are shown in Figure 2. $\mathrm{LUT}_{\text {SECT }}$ and $\mathrm{LUT}_{77 \mathrm{keV}}$ formed a bilinear relation between the HU value and theoretical SPR, while LUT $_{140 \mathrm{keV}}$ showed an almost linear relation $\left(r^{2}=0.989\right)$. SPR $_{\mathrm{WD}}$ provided the highest linear relation with the theoretical SPR $\left(r^{2}=0.998\right)$.

Figure 3 shows the percentage difference between $S_{\text {PPD }}$ and theoretical SPR for each reference material. The overall mean difference was $-0.3 \%$, and the mean difference was below $2 \%$ for most reference materials. LN-300 lung provided the largest difference of $5.4 \%$, while the difference was $-4.3 \%$ for BRN-SR2 brain, indicating a large difference

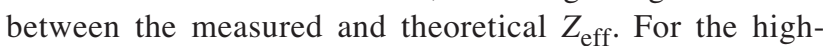
density reference material of SB3 cortical bone, the difference was $-3.3 \%$.

Figure 4 shows the absolute percentage difference between the measured $\left(\mathrm{SPR}_{\mathrm{SECT}}, \mathrm{SPR}_{77 \mathrm{keV}}, \mathrm{SPR}_{140 \mathrm{keV}}\right.$,
$\mathrm{SPR}_{\mathrm{WD}}$ ) and theoretical SPRs for different phantom sizes. For the large phantom, a large difference was observed for low-density reference materials (LN-300 and 450 lung), with the largest difference being $29.9 \%$ and $32.2 \%$ for $\mathrm{SPR}_{\mathrm{SECT}}$ and $\mathrm{SPR}_{77 \mathrm{keV}}$, respectively. The differences in $\mathrm{SPR}_{140 \mathrm{keV}}$ and $\mathrm{SPR}_{\mathrm{WD}}$ reduced to $19.7 \%$ and $19.3 \%$, respectively. Similarly, the differences in $\mathrm{SPR}_{140 \mathrm{keV}}(2.8 \%)$ and $\mathrm{SPR}_{\mathrm{WD}}(5.8 \%)$ for the high-density reference material of SB3 cortical bone were smaller than those in SPR SECT $_{1}$ $(11.2 \%)$ and $\operatorname{SPR}_{77 \mathrm{keV}}(10.7 \%)$. As a result, the mean absolute difference in $\operatorname{SPR}_{140 \mathrm{keV}}(3.0 \%, p=0.006)$ and $\mathrm{SPR}_{\mathrm{WD}}(3.2 \%, p=0.002)$ for the 16 reference materials was significantly lower than that in $\mathrm{SPR}_{\mathrm{SECT}}$, which used conventional SECT (5.9\%). For the small phantom, the difference was below $5 \%$ for most reference materials. Regarding $\mathrm{SPR}_{140 \mathrm{keV}}$, the maximum difference was $3.1 \%$ for SB3 cortical bone. A significant reduction in the mean difference for the SPR calculation was observed in $\operatorname{SPR}_{77 \mathrm{keV}}(1.0 \%, p=0.001)$ and $\operatorname{SPR}_{140 \mathrm{keV}}(1.1 \%, p=0.013)$ compared with $\mathrm{SPR}_{\mathrm{SECT}}(2.2 \%)$.

\section{Discussion}

In this study, we evaluated the accuracy of SPR calculation based on the SECT, $\mathrm{VMI}_{77 \mathrm{keV}}, \mathrm{VMI}_{140 \mathrm{keV}}$, and WDI 


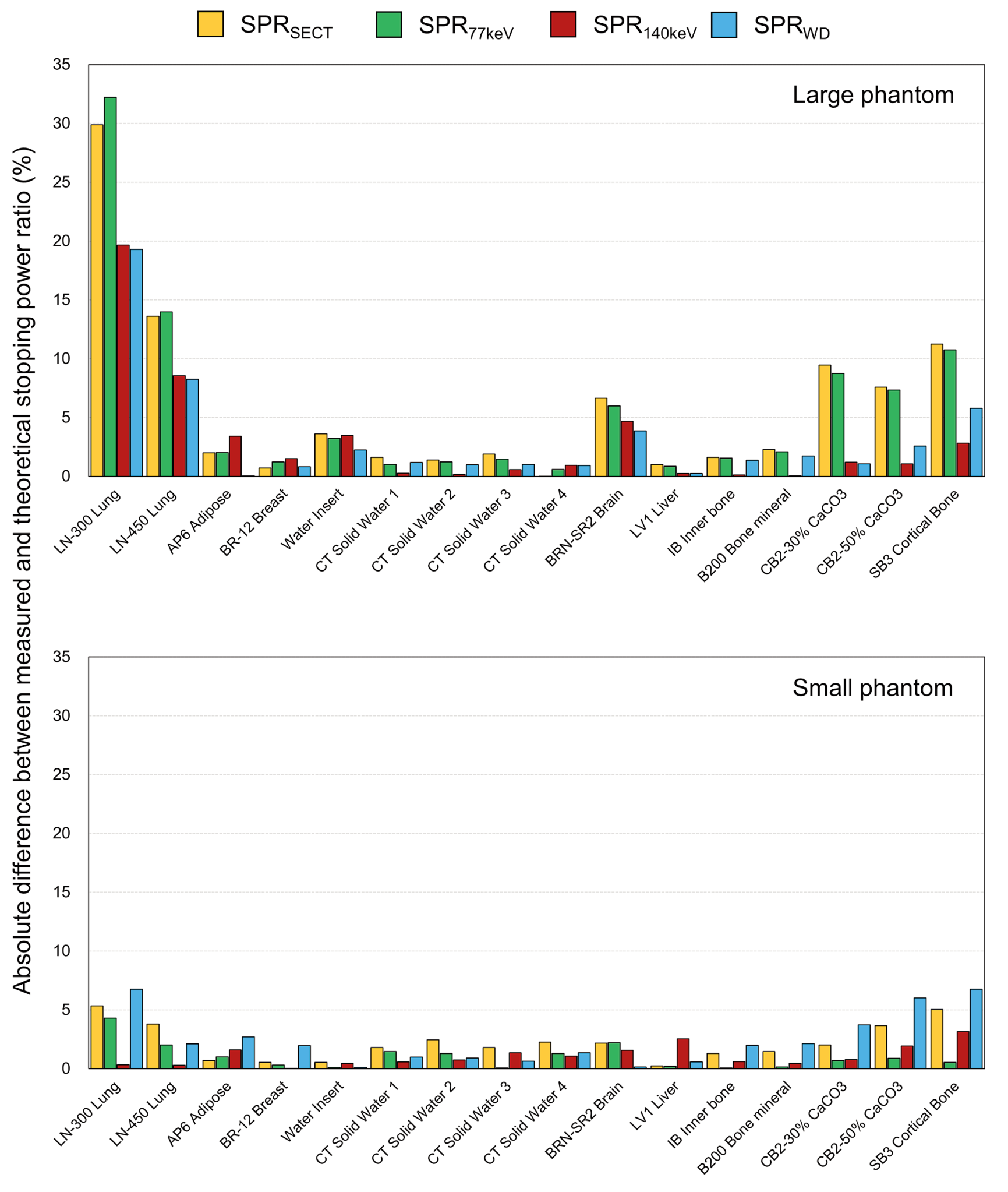

\section{Reference materials}

Figure 4. Absolute percentage difference between measured $\left(S P R_{S E C T}, S P R_{77 k e V}, S P R_{140 \mathrm{keV}}\right.$ and $\left.S P R_{W D}\right)$ and theoretical stopping power ratios (SPRs) for small and large phantoms. 
generated using FKSCT for different phantom sizes. For treatment planning of particle therapy, a piecewise linear function obtained using a tissue-mimicking phantom is usually applied to convert HU values obtained from SECT into the corresponding SPRs. As X-rays in CT scanners contain polychromatic energies, beam hardening, which induces inaccurate $\mathrm{HU}$ values under the presence of highdensity materials and depending on the size of the scanned object, is unavoidable (14). Thus, it is difficult to improve the accuracy of SPR calculation to reduce the uncertainty in dose calculation for particle therapy. Taasti et al. found that particle therapy institutions recognize DECT as a highly important emerging technique to improve range prediction accuracy (15).

DECT is being increasingly applied to clinical radiotherapy to generate VMIs at a given energy level (commonly from 40 to $140 \mathrm{keV}$ ), and the VMI at $70-79 \mathrm{keV}$ can be considered as the equivalent effective energy of 120 $\mathrm{kVp}$ polychromatic X-ray imaging $(16,17)$. In our study, $\mathrm{VMI}_{77 \mathrm{keV}}$ provided almost the same HU values from SECT images for various reference materials, and $\mathrm{VMI}_{140 \mathrm{keV}}$ showed a linear relation between the HU values and theoretical SPRs (Figure 2). As the Compton effect is dominant at high energy levels, $\mathrm{VMI}_{140 \mathrm{keV}}$ is less affected by beam hardening and provides reliable HU values. In this study, we first reported the linear relation between $S P R_{W D}$, which is generated from WDI and $Z_{\text {eff }}$, and the theoretical SPR, obtaining an overall mean difference of $-0.3 \%$. A similar accuracy has been reported using dual-layer CT (overall mean difference of $0.3 \%$ ) (6) and dual-source CT $(0.6 \%)(12)$. Such accuracy in SPR measurements can in turn improve the accuracy of dose calculation for particle therapy.

Taasti et al. reported that 7 out of 12 particle therapy institutions use from 2 to 12 LUTs (e.g., for head, body, and children) for dose calculation to minimize the effect of inaccurate conversion from $\mathrm{HU}$ values to SPRs, while 5 institutions use a single LUT for all treatment sites (15). As complicated treatment planning workflow involving multiple LUTs can induce errors, a simple workflow with a single conversion method would be ideal in clinical practice. In this study, we evaluated the accuracy of SPR calculation for small and large phantoms, obtaining inaccurate results in the large phantom when using SECT and $\mathrm{VMI}_{77 \mathrm{keV}}$ to generate the LUT (Figure 4). For patients with prostate cancer, heavy particle therapy may be ideal given its shape dosimetric features and biological effect with linear energy transfer (1). Okada et al. reported that the hypofractionated heavy particle therapy regimen provides a lower incidence of genitourinary toxicity and similar biochemical relapse-free survival compared with conventional fractionation (18). For such sophisticated treatment, an unexpected radiation side effect may occur under inaccurate dose calculation, while obesity has been linked to an increased risk of prostate cancer progression (19). The conversion from HU values to SPRs using conventional SECT may not be suitable for large patients, while $\mathrm{VMI}_{140 \mathrm{keV}}$ and WDI may improve the accuracy of dose calculation. For the small phantom in this study, $\mathrm{VMI}_{77 \mathrm{keV}}$ and $\mathrm{VMI}_{140 \mathrm{keV}}$ provided significantly smaller differences in SPR calculation than SECT (Figure 4). Therefore, $\mathrm{VMI}_{140 \mathrm{keV}}$ seems to be the most robust method for conversion from $\mathrm{HU}$ values to SPRs when using FKSCT for treatment planning of particle therapy.

Various limitations of this study should be noted. First, only one acquisition setting was used for phantom scanning, but measurements vary depending on scanning parameters such as radiation dose, reconstruction kernels, and the use of iterative reconstruction (5). Second, we used a tissuemimicking phantom, and human anatomical features could not be considered. Third, the accuracy of SPR calculation using FKSCT was evaluated, and direct comparisons with those obtained using dual-source CT and dual-layer CT were not performed. Finally, the effect of the improved SPR calculation using FKSCT on the dose calculation accuracy for particle therapy remains to be investigated. Despite these limitations, we believe that our quantitative analyses can provide useful information for treatment planning of particle therapy to reduce the uncertainty in the conversion from $\mathrm{HU}$ values to SPRs.

In conclusion, $\mathrm{VMI}_{140 \mathrm{keV}}$ and WDI generated using FKSCT provide a more linear relation between the measured values and theoretical SPR than those generated using SECT. For large and small phantoms, $\mathrm{VMI}_{140 \mathrm{keV}}$ significantly improves the SPR calculation compared with SECT-based calculation. The robust SPR calculation using FKSCT has the potential to improve the dose calculation accuracy in treatment planning of particle therapy.

\section{Funding}

This study was supported by a JSPS KAKENHI Grant [Grant-inAid for Young Scientists 19K17285 and Grant-in-Aid for Scientific Research (C) 21K07742].

\section{Conflicts of Interest}

The Authors have no conflicts of interest to disclose in relation to this study.

\section{Authors' Contributions}

Research design: OS; experimental work: OS, IY; statistical analysis: OS; manuscript writing: OS, IY, KY, OS, UY, MM, KM, KK.

\section{Acknowledgements}

The Authors would like to thank Editage (www.editage.com) for English language editing. 


\section{References}

1 Malouff TD, Mahajan A, Krishnan S, Beltran C, Seneviratne DS and Trifiletti DM: Carbon ion therapy: a modern review of an emerging technology. Front Oncol 10: 82, 2020. PMID: 32117737. DOI: $10.3389 /$ fonc. 2020.00082

2 Wohlfahrt P, Möhler C, Stützer K, Greilich S and Richter C: Dual-energy CT based proton range prediction in head and pelvic tumor patients. Radiother Oncol 125(3): 526-533, 2017. PMID: 29050953. DOI: 10.1016/j.radonc.2017.09.042

3 Zurl B, Tiefling R, Winkler P, Kindl $\mathrm{P}$ and Kapp KS: Hounsfield units variations: impact on CT-density based conversion tables and their effects on dose distribution. Strahlenther Onkol 190(1): 88-93, 2014. PMID: 24201381. DOI: $10.1007 / \mathrm{s} 00066-013-0464-5$

4 Ohira S, Komiyama R, Karino T, Washio H, Ueda Y, Miyazaki M, Koizumi $\mathbf{M}$ and Teshima T: Volumetric modulated arc therapy planning based on virtual monochromatic images: Effect of inaccurate CT numbers on dose distributions. Phys Med 60: 83-90, 2019. PMID: 31000091. DOI: 10.1016/j.ejmp.2019. 03.022

5 Ohira S, Karino T, Ueda Y, Nitta Y, Kanayama N, Miyazaki M, Koizumi M and Teshima T: How well does dual-energy CT with fast kilovoltage switching quantify CT number and iodine and calcium concentrations? Acad Radiol 25(4): 519-528, 2018. PMID: 29275940. DOI: 10.1016/j.acra.2017.11.002

6 Ohira S, Washio H, Yagi M, Karino T, Nakamura K, Ueda Y, Miyazaki M, Koizumi M and Teshima T: Estimation of electron density, effective atomic number and stopping power ratio using dual-layer computed tomography for radiotherapy treatment planning. Phys Med 56: 34-40, 2018. PMID: 30527087. DOI: 10.1016/j.ejmp.2018.11.008

7 Polf JC, Mille MM, Mossahebi S, Chen H, Maggi P and ChenMayer $\mathrm{H}$ : Determination of proton stopping power ratio with dual-energy CT in 3D-printed tissue/air cavity surrogates. Med Phys 46(7): 3245-3253, 2019. PMID: 31081542. DOI: 10.1002/ mp. 13587

8 Johnson TR: Dual-energy CT: general principles. AJR Am J Roentgenol 199(5 Suppl): S3-S8, 2012. PMID: 23097165. DOI: 10.2214/AJR.12.9116

9 Goodsitt MM, Christodoulou EG and Larson SC: Accuracies of the synthesized monochromatic CT numbers and effective atomic numbers obtained with a rapid $\mathrm{kVp}$ switching dual energy CT scanner. Med Phys 38(4): 2222-2232, 2011. PMID: 21626956. DOI: $10.1118 / 1.3567509$

10 Ohira S, Yagi M, Iramina H, Karino T, Washio H, Ueda Y, Miyazaki M, Koizumi M and Teshima T: Treatment planning based on water density image generated using dual-energy computed tomography for pancreatic cancer with contrastenhancing agent: Phantom and clinical study. Med Phys 45(11): 5208-5217, 2018. PMID: 30198189. DOI: 10.1002/mp.13180

11 Landry G, Seco J, Gaudreault M and Verhaegen F: Deriving effective atomic numbers from DECT based on a parameterization of the ratio of high and low linear attenuation coefficients. Phys Med Biol 58(19): 6851-6866, 2013. PMID: 24025623. DOI: $10.1088 / 0031-9155 / 58 / 19 / 6851$
12 Hünemohr N, Krauss B, Tremmel C, Ackermann B, Jäkel O and Greilich S: Experimental verification of ion stopping power prediction from dual energy CT data in tissue surrogates. Phys Med Biol 59(1): 83-96, 2014. PMID: 24334601. DOI: 10.1088/ 0031-9155/59/1/83

13 Berger MJ, Hubbell JH, Seltzer SM, Chang J, Coursey JS, Sukumar R, Zucker DS and Olsen K: Xcom: Photon cross sections database. NIST Standard Reference Database 8 (XGAM). Available at: https://www.nist.gov/pml/xcom-photoncross-sections-database Last Update to Data Content: November 2010. [Last accessed on October 29, 2021]

14 Barrett JF and Keat N: Artifacts in CT: recognition and avoidance. Radiographics 24(6): 1679-1691, 2004. PMID: 15537976. DOI: $10.1148 / \mathrm{rg} .246045065$

15 Taasti VT, Bäumer C, Dahlgren CV, Deisher AJ, Ellerbrock M, Free J, Gora J, Kozera A, Lomax AJ, De Marzi L, Molinelli S, Kevin Teo BK, Wohlfahrt P, Petersen JBB, Muren LP, Hansen DC and Richter C: Inter-centre variability of CT-based stoppingpower prediction in particle therapy: Survey-based evaluation. Phys Imaging Radiat Oncol 6: 25-30, 2018. PMID: 33458385. DOI: $10.1016 /$ j.phro.2018.04.006

16 Yagi M, Ueguchi T, Koizumi M, Ogata T, Yamada S, Takahashi Y, Sumida I, Akino Y, Konishi K, Isohashi F, Tomiyama N, Yoshioka $\mathrm{Y}$ and Ogawa $\mathrm{K}$ : Gemstone spectral imaging: determination of $\mathrm{CT}$ to $\mathrm{ED}$ conversion curves for radiotherapy treatment planning. J Appl Clin Med Phys 14(5): 173-186, 2013. PMID: 24036870. DOI: 10.1120/jacmp.v14i5.4335

17 Wohlfahrt P, Möhler C, Hietschold V, Menkel S, Greilich S, Krause M, Baumann M, Enghardt W and Richter C: Clinical implementation of dual-energy CT for proton treatment planning on pseudo-monoenergetic CT scans. Int J Radiat Oncol Biol Phys 97(2): 427-434, 2017. PMID: 28068248. DOI: 10.1016/ j.ijrobp.2016.10.022

18 Okada T, Tsuji H, Kamada T, Akakura K, Suzuki H, Shimazaki J, Tsujii $\mathrm{H}$ and Working Group for Genitourinary Tumors: Carbon ion radiotherapy in advanced hypofractionated regimens for prostate cancer: from 20 to 16 fractions. Int J Radiat Oncol Biol Phys 84(4): 968-972, 2012. PMID: 22898380. DOI: 10.1016/j.ijrobp.2012.01.072

19 Labbé DP, Zadra G, Yang M, Reyes JM, Lin CY, Cacciatore S, Ebot EM, Creech AL, Giunchi F, Fiorentino M, Elfandy H, Syamala S, Karoly ED, Alshalalfa M, Erho N, Ross A, Schaeffer EM, Gibb EA, Takhar M, Den RB, Lehrer J, Karnes RJ, Freedland SJ, Davicioni E, Spratt DE, Ellis L, Jaffe JD, D'Amico AV, Kantoff PW, Bradner JE, Mucci LA, Chavarro JE, Loda $\mathrm{M}$ and Brown $\mathrm{M}$ : High-fat diet fuels prostate cancer progression by rewiring the metabolome and amplifying the MYC program. Nat Commun 10(1): 4358, 2019. PMID: 31554818. DOI: 10.1038/s41467-019-12298-Z

Received October 1, 2021

Revised October 28, 2021

Accepted October 29, 2021 